Supplementary Material for

Heptahexaenylidene Complexes: Synthesis and Characterization of the First Complexes with an $\mathrm{M}=\mathrm{C}=\mathrm{C}=\mathrm{C}=\mathrm{C}=\mathrm{C}=\mathrm{C}=\mathrm{CR}_{2}$ Moiety $(\mathrm{M}=\mathrm{Cr}, \mathrm{W})$

Markus Dede, Matthias Drexler, and Helmut Fischer

Fachbereich Chemie, Universität Konstanz, Fach M727, 78457 Konstanz, Germany

Tables giving the calculated Cartesian coordinates, bond distances, bond angles and energies of complexes 5a and 8a, breakdown of the orbital contributions of the metal and the carbon atoms along the chain to the HOMO and LUMO of these complexes. 
$(\mathrm{CO})_{5} \mathrm{Cr}=\mathrm{C}=\mathrm{C}=\mathrm{C}=\mathrm{C}=\mathrm{C}=\mathrm{C}=\mathrm{C}\left(\mathrm{NMe}_{2}\right)_{2} \quad$ (5a)

energy: $\quad-1188.84250142345$ hartrees $\quad-746011 \mathrm{kcal} / \mathrm{mol} \quad-32351 \mathrm{eV}$

\section{Frontier orbitals}

90 Orbital Energy $\quad-0.081537$ Occupation $0.00 \quad-51.17 \mathrm{kcal} / \mathrm{mol} \quad-2.219 \mathrm{eV}$

89 Orbital Energy $\quad-0.122381$ Occupation $0.00 \quad-76.79 \mathrm{kcal} / \mathrm{mol} \quad-3.330 \mathrm{eV}$

88 Orbital Energy $\quad-0.168788$ Occupation $1.00 \quad-105.92 \mathrm{kcal} / \mathrm{mol} \quad-4.593 \mathrm{eV}$

87 Orbital Energy $\quad-0.177551$ Occupation $1.00 \quad-111.42 \mathrm{kcal} / \mathrm{mol} \quad-4.832 \mathrm{eV}$

Breakdown of the orbital contributions of chromium and the carbon atoms along the chain to the HOMO and LUMO of $(\mathrm{CO})_{5} \mathrm{Cr}=\mathrm{C} 1=\mathrm{C} 2=\mathrm{C} 3=\mathrm{C} 4=\mathrm{C} 5=\mathrm{C} 6=\mathrm{C} 7\left(\mathrm{NMe}_{2}\right)_{2}$

$\begin{array}{rrrrrrrrr} & \text { Cr } & \text { C24 } & \text { C25 } & \text { C21 } & \text { C36 } & \text { C35 } & \text { C4 } & \text { C5 } \\ \text { HOMO } & 33.1 & 5.4 & 15.3 & 1.1 & 14.1 & 0.4 & 11.4 & 0.7 \\ \text { LUMO } & 5.9 & 14.0 & 2.2 & 15.2 & 0.7 & 17.5 & 1.7 & 18.6\end{array}$

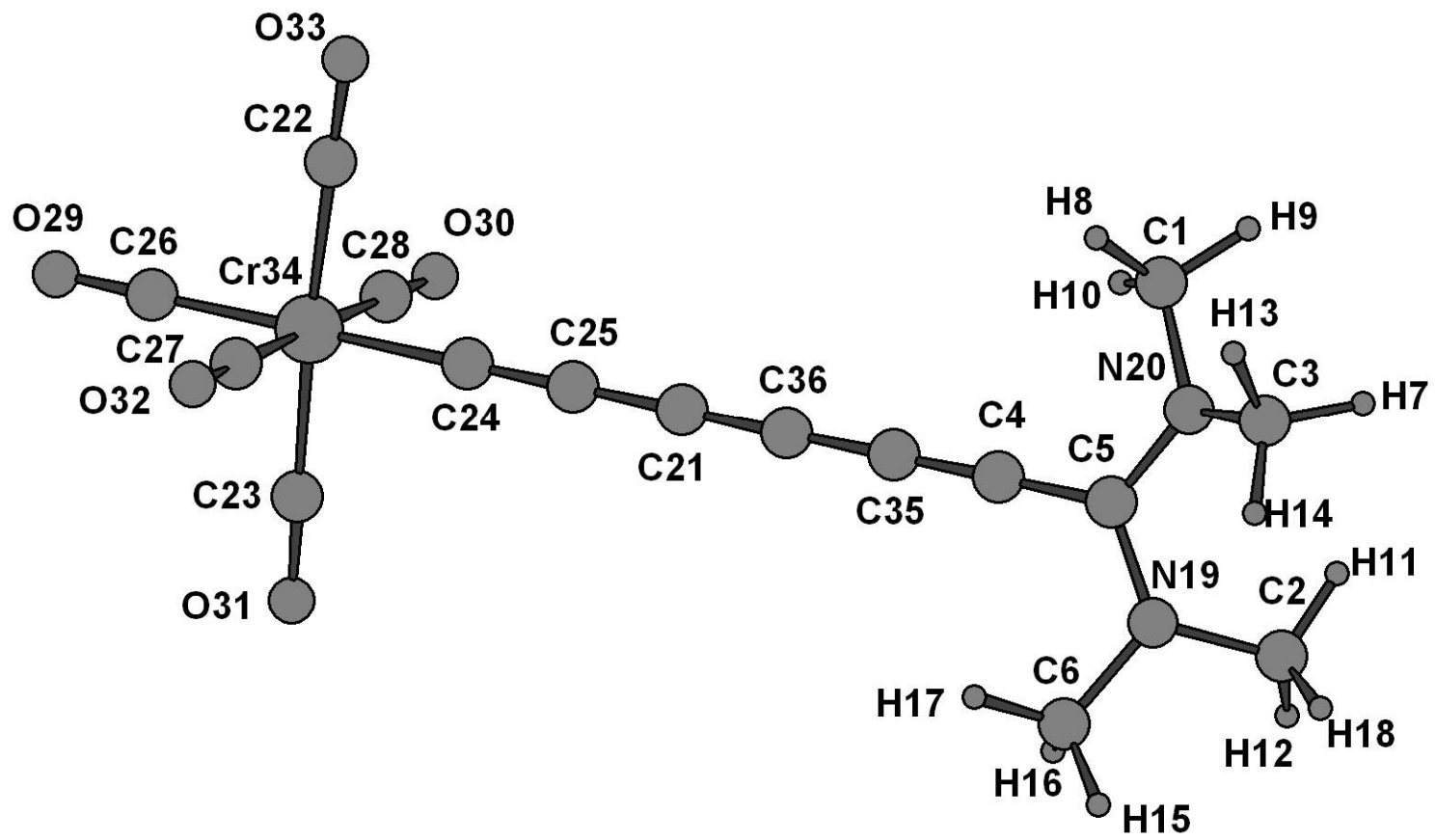




\section{Coordinates}

\begin{tabular}{|c|c|c|c|}
\hline atom & $\mathrm{x}$ & y & z \\
\hline $\mathrm{C} 1$ & 0.5457226390 & 2.3204100274 & 6.2219746296 \\
\hline $\mathrm{C} 2$ & -0.2856250489 & -1.4037096340 & 8.1329768982 \\
\hline $\mathrm{C} 3$ & -0.9036200341 & 1.6124382126 & 8.0923479239 \\
\hline $\mathrm{C} 4$ & -0.4149348739 & 0.0887257312 & 4.7851040743 \\
\hline $\mathrm{C} 5$ & -0.4932400951 & 0.0978140325 & 6.1598248762 \\
\hline $\mathrm{C} 6$ & -1.5409005245 & -2.1207594324 & 6.1327614293 \\
\hline $\mathrm{H} 7$ & -0.2359328972 & 1.7416616980 & 8.9648784574 \\
\hline H8 & -0.1623258699 & 3.0153823669 & 5.7260054141 \\
\hline $\mathrm{H} 9$ & 1.1271101066 & 2.8827141384 & 6.9747798431 \\
\hline $\mathrm{H} 10$ & 1.2319205007 & 1.9156786318 & 5.4625456983 \\
\hline $\mathrm{H} 11$ & 0.4136538760 & -0.6217131556 & 8.4617027159 \\
\hline $\mathrm{H} 12$ & -1.0411836529 & -1.5720597322 & 8.9224937755 \\
\hline $\mathrm{H} 13$ & -1.4210370938 & 2.5731382237 & 7.9070656102 \\
\hline $\mathrm{H} 14$ & -1.6610801936 & 0.8508206970 & 8.3265428525 \\
\hline H1 5 & -2.1772928986 & -2.6908139079 & 6.8314484225 \\
\hline $\mathrm{H} 16$ & -0.7853633164 & -2.8068719228 & 5.6990417736 \\
\hline $\mathrm{H} 17$ & -2.1638710390 & -1.7237904111 & 5.3172722281 \\
\hline H1 8 & 0.2845642698 & -2.3429828291 & 7.9923590059 \\
\hline N19 & -0.9170461138 & -1.0170324745 & 6.8656812827 \\
\hline N20 & -0.1532612253 & 1.2231373777 & 6.8925858044 \\
\hline C21 & -0.2086082438 & 0.0529857939 & 0.9405390214 \\
\hline C22 & 0.3182000889 & 1.8864750948 & -3.5044563318 \\
\hline C23 & -0.2670629912 & -1.8466229729 & -3.5101096913 \\
\hline C24 & -0.0708112478 & 0.0328345872 & -1.6483826388 \\
\hline C25 & -0.1384662605 & 0.0423086733 & -0.3744066306 \\
\hline C26 & 0.1235679373 & 0.0079549571 & -5.4615542438 \\
\hline C27 & -1.8410807103 & 0.3155021312 & -3.6369726370 \\
\hline C28 & 1.8952975713 & -0.2767358602 & -3.4434470707 \\
\hline 029 & 0.1810573040 & 0.0014879640 & -6.6284174724 \\
\hline 030 & 3.0431964112 & -0.4589961707 & -3.3537681636 \\
\hline 031 & -0.4497406732 & -2.9971320017 & -3.4562359467 \\
\hline 032 & -2.9922134021 & 0.4971961613 & -3.6661561570 \\
\hline 033 & 0.4954237112 & 3.0376638895 & -3.4473501622 \\
\hline $\operatorname{Cr} 34$ & 0.0286027080 & 0.0195622156 & -3.5694797944 \\
\hline C35 & -0.3478972872 & 0.0749685672 & 3.5234113506 \\
\hline C36 & -0.2761674981 & 0.0629381705 & 2.2084603682 \\
\hline
\end{tabular}




\section{bond lengths (angstroms):}

\begin{tabular}{|c|c|c|c|}
\hline $\mathrm{C} 1-\mathrm{H} 8$ & 1.109191 & $\mathrm{C} 1-\mathrm{H} 9$ & 1.104949 \\
\hline $\mathrm{C} 1-\mathrm{H} 10$ & 1.100640 & $\mathrm{C} 1-\mathrm{N} 20$ & 1.463662 \\
\hline $\mathrm{C} 2-\mathrm{H} 11$ & 1.099350 & $\mathrm{C} 2-\mathrm{H} 12$ & 1.105689 \\
\hline $\mathrm{C} 2-\mathrm{H} 18$ & 1.107756 & $\mathrm{C} 2-\mathrm{N} 19$ & 1.467736 \\
\hline $\mathrm{C} 3-\mathrm{H} 7$ & 1.106261 & $\mathrm{C} 3-\mathrm{H} 13$ & 1.106795 \\
\hline $\mathrm{C} 3-\mathrm{H} 14$ & 1.099388 & $\mathrm{C} 3-\mathrm{N} 20$ & 1.467659 \\
\hline $\mathrm{C} 4-\mathrm{C} 5$ & 1.376979 & C4 -C35 & 1.263547 \\
\hline C5 -N19 & 1.385903 & $\mathrm{C} 5-\mathrm{N} 20$ & 1.385235 \\
\hline $\mathrm{C} 6-\mathrm{H} 15$ & 1.103685 & $\mathrm{C} 6-\mathrm{H} 16$ & 1.108918 \\
\hline $\mathrm{C} 6-\mathrm{H} 17$ & 1.100318 & $\mathrm{C} 6-\mathrm{N} 19$ & 1.464438 \\
\hline $\mathrm{C} 21-\mathrm{C} 25$ & 1.316858 & $\mathrm{C} 21-\mathrm{C} 36$ & 1.269759 \\
\hline $\mathrm{C} 22-033$ & 1.166150 & C22-Cr34 & 1.890359 \\
\hline $\mathrm{C} 23-031$ & 1.166167 & C23-Cr34 & 1.890394 \\
\hline $\mathrm{C} 24-\mathrm{C} 25$ & 1.275806 & C24-Cr34 & 1.923713 \\
\hline $\mathrm{C} 26-029$ & 1.168296 & C26-Cr34 & 1.894492 \\
\hline $\mathrm{C} 27-032$ & 1.165749 & C27-Cr34 & 1.894163 \\
\hline $\mathrm{C} 28-030$ & 1.165733 & $\mathrm{C} 28-\operatorname{Cr} 34$ & 1.894261 \\
\hline
\end{tabular}

C35-C36 1.316961 


\section{bond angles:}

\begin{tabular}{|c|c|c|c|c|}
\hline H9 & $-\mathrm{C} 1$ & $-\mathrm{H} 8$ & : & 108.763665 \\
\hline 10 & $-\mathrm{C} 1$ & $-\mathrm{H} 9$ & : & 109.219252 \\
\hline 20 & $-\mathrm{C} 1$ & $-\mathrm{H} 9$ & : & 108.700877 \\
\hline 2 & $-\mathrm{C} 2$ & $-\mathrm{H} 11$ & $:$ & 109.235475 \\
\hline 8 & $-\mathrm{C} 2$ & $-\mathrm{H} 12$ & $:$ & 108.256475 \\
\hline 9 & $-\mathrm{C} 2$ & $-\mathrm{H} 12$ & $:$ & 111.264653 \\
\hline 3 & $-\mathrm{C} 3$ & $-\mathrm{H} 7$ & $:$ & 108.227981 \\
\hline-4 & $-\mathrm{C} 3$ & $-\mathrm{H} 13$ & $:$ & 108.354080 \\
\hline 20 & $-\mathrm{C} 3$ & $-\mathrm{H} 13$ & $:$ & 109.414716 \\
\hline 35 & $-\mathrm{C} 4$ & $-\mathrm{C} 5$ & $:$ & 179.671126 \\
\hline 0 & $-\mathrm{C} 5$ & $-\mathrm{C} 4$ & $:$ & 121.299833 \\
\hline 6 & $-\mathrm{C} 6$ & $-\mathrm{H} 15$ & $:$ & 108.716497 \\
\hline-7 & $-\mathrm{C} 6$ & $-\mathrm{H} 16$ & $:$ & 108.608271 \\
\hline 19 & $-\mathrm{C} 6$ & $-\mathrm{H} 16$ & $:$ & 111.828028 \\
\hline & $-\mathrm{N} 19$ & $-\mathrm{C} 2$ & $:$ & 121.340870 \\
\hline & $-\mathrm{N} 19$ & $-\mathrm{C} 5$ & $:$ & 118.793258 \\
\hline & $-\mathrm{N} 20$ & $-\mathrm{C} 1$ & $:$ & 118.937645 \\
\hline 36 & $-\mathrm{C} 21$ & $-\mathrm{C} 25$ & $:$ & 179.984170 \\
\hline 234 & $-\mathrm{C} 23$ & -031 & $:$ & 179.151665 \\
\hline 4 & $-\mathrm{C} 25$ & $-\mathrm{C} 21$ & $:$ & 179.958646 \\
\hline CR34 & $-\mathrm{C} 27$ & -032 & $:$ & 179.391997 \\
\hline 3 & $-\operatorname{Cr} 34$ & $-\mathrm{C} 22$ & $:$ & 176.224501 \\
\hline 24 & $-\operatorname{Cr} 34$ & $-C 23$ & $:$ & 88.129810 \\
\hline 26 & $-\operatorname{Cr} 34$ & $-C 23$ & : & 91.900152 \\
\hline 27 & $-\operatorname{Cr} 34$ & $-\mathrm{C} 22$ & $:$ & 89.893590 \\
\hline 27 & $-\operatorname{Cr} 34$ & $-C 24$ & $:$ & 89.054309 \\
\hline 28 & $-\operatorname{Cr} 34$ & $-\mathrm{C} 22$ & : & 90.070046 \\
\hline C28 & $-\operatorname{Cr} 34$ & $-C 24$ & : & 89.172724 \\
\hline & $-\operatorname{Cr} 34$ & $-\mathrm{C} 27$ & $:$ & 178.226889 \\
\hline & $-\mathrm{C} 36$ & $-\mathrm{C} 21$ & : & 9.8 \\
\hline
\end{tabular}

\begin{tabular}{|c|c|c|c|c|}
\hline $\mathrm{H} 10$ & $-\mathrm{C} 1$ & $-\mathrm{H} 8$ & : & 108.654152 \\
\hline 120 & $-\mathrm{C} 1$ & $-\mathrm{H} 8$ & $:$ & 111.699454 \\
\hline 20 & $-\mathrm{C} 1$ & $-\mathrm{H} 10$ & $:$ & 109.767025 \\
\hline 18 & $-\mathrm{C} 2$ & $-\mathrm{H} 11$ & $:$ & 108.281571 \\
\hline 19 & $-\mathrm{C} 2$ & $-\mathrm{H} 11$ & $:$ & 110.146817 \\
\hline 19 & $-\mathrm{C} 2$ & $-\mathrm{H} 18$ & : & 109.585486 \\
\hline 4 & $-\mathrm{C} 3$ & $-\mathrm{H} 7$ & : & 09.192612 \\
\hline 20 & $-\mathrm{C} 3$ & $-\mathrm{H} 7$ & : & 111.540752 \\
\hline 20 & $-\mathrm{C} 3$ & $-\mathrm{H} 14$ & : & 110.037343 \\
\hline 19 & $-\mathrm{C} 5$ & $-\mathrm{C} 4$ & : & 121.369608 \\
\hline 20 & $-\mathrm{C} 5$ & $-\mathrm{N} 19$ & : & 117.330486 \\
\hline 17 & $-\mathrm{C} 6$ & $-\mathrm{H} 15$ & : & 109.211734 \\
\hline 19 & $-\mathrm{C} 6$ & $-\mathrm{H} 15$ & : & 108.547345 \\
\hline J19 & $-\mathrm{C} 6$ & $-\mathrm{H} 17$ & : & 109.889262 \\
\hline C 6 & $-\mathrm{N} 19$ & $-\mathrm{C} 2$ & : & 114.635104 \\
\hline C3 & $-\mathrm{N} 20$ & $-\mathrm{C} 1$ & : & 114.824829 \\
\hline C5 & $-\mathrm{N} 20$ & $-\mathrm{C} 3$ & : & 121.495199 \\
\hline ה & $\mathrm{A}-\mathrm{C} 22$ & -033 & : & 179.161759 \\
\hline 534 & -C24 & $-\mathrm{C} 25$ & : & 179.916807 \\
\hline 4 & t $-\mathrm{C} 26$ & -029 & : & 179.937312 \\
\hline $\operatorname{Cr} 34$ & $\mathrm{t}-\mathrm{C} 28$ & -030 & : & 179.402849 \\
\hline C24 & $-\operatorname{Cr} 34$ & $-\mathrm{C} 22$ & : & 88.094709 \\
\hline C26 & $-\operatorname{Cr} 34$ & $-\mathrm{C} 22$ & : & 91.875343 \\
\hline C26 & $-\operatorname{Cr} 34$ & $-C 24$ & : & 179.900598 \\
\hline C27 & $-\operatorname{Cr} 34$ & $-C 23$ & : & 90.055756 \\
\hline C27 & $-\operatorname{Cr} 34$ & $-C 26$ & : & 90.850868 \\
\hline $\mathrm{C} 28$ & $-\operatorname{Cr} 34$ & $-C 23$ & : & 89.863804 \\
\hline C28 & $-\operatorname{Cr} 34$ & $-C 26$ & : & 90.922111 \\
\hline & $-\mathrm{C} 35$ & $-\mathrm{C} 4$ & : & 79.8710 \\
\hline
\end{tabular}


$(\mathrm{CO})_{5} \mathrm{Cr}=\mathrm{C}=\mathrm{C}=\mathrm{C}=\mathrm{C}=\mathrm{C}=\mathrm{C}=\mathrm{C}(\mathrm{Me}) \mathrm{NMe}_{2}$ (8a)

energy: $\quad-1094.1881$ hartrees $\quad-686613 \mathrm{kcal} / \mathrm{mol} \quad-29775 \mathrm{eV}$

\section{Frontier orbitals}

82 Orbital Energy $\quad-0.084374$ Occupation $0.00 \quad-52.9455 \mathrm{kcal} / \mathrm{mol} \quad-2.2960 \mathrm{eV}$

81 Orbital Energy $\quad-0.131149$ Occupation $0.00 \quad-82.2973 \mathrm{kcal} / \mathrm{mol} \quad-3.5688 \mathrm{eV}$

80 Orbital Energy $\quad-0.173247$ Occupation $1.00 \quad-108.7142 \mathrm{kcal} / \mathrm{mol} \quad-4.7144 \mathrm{eV}$

79 Orbital Energy $\quad-0.183412$ Occupation $1.00 \quad-115.0929 \mathrm{kcal} / \mathrm{mol} \quad-4.9910 \mathrm{eV}$

Contributions of chromium and the various $\mathrm{C}$ atoms of the chain to the HOMO and LUMO of $(\mathrm{CO})_{5} \mathrm{Cr}=\mathrm{C} 1=\mathrm{C} 2=\mathrm{C} 3=\mathrm{C} 4=\mathrm{C} 5=\mathrm{C} 6=\mathrm{C} 7\left(\mathrm{NMe}_{2}\right) \mathrm{Me}$

$\begin{array}{rrrrrrrrr} & \text { Cr27 } & \text { C10 } & \text { C9 } & \text { C13 } & \text { C14 } & \text { C5 } & \text { C2 } & \text { C3 } \\ \text { HOMO } & 31.3 & 6.2 & 15.6 & 1.4 & 14.7 & 0.5 & 12.2 & 0.6 \\ \text { LUMO } & 6.7 & 13.6 & 2.8 & 14.6 & 1.0 & 17.0 & 0.9 & 21.2\end{array}$

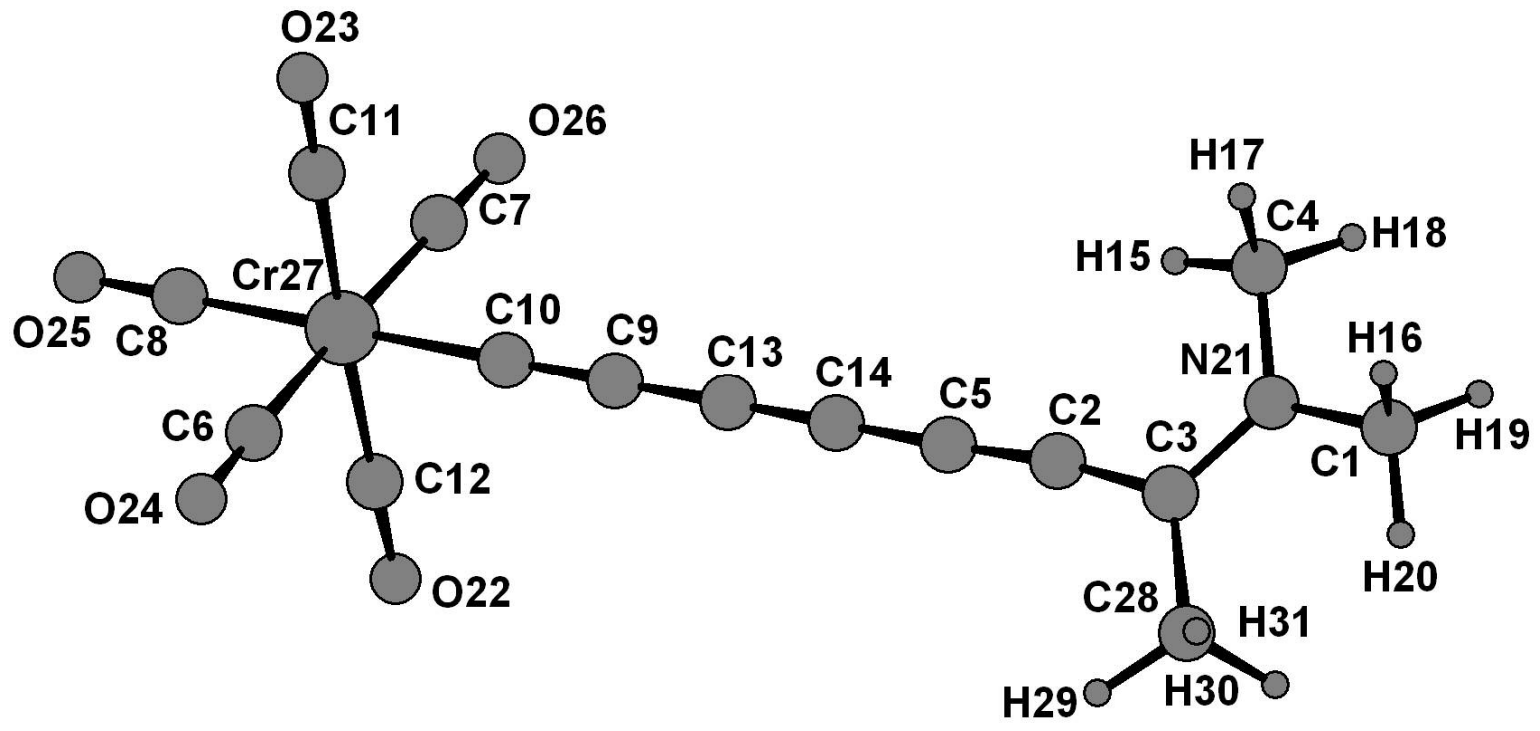




\section{Atom coordinates}

\begin{tabular}{|c|c|}
\hline atom & $\mathrm{x}$ \\
\hline $\mathrm{C} 1$ & -0.944369016 \\
\hline $\mathrm{C} 2$ & -0.32579194 \\
\hline C3 & -0.29789169 \\
\hline C4 & -1.80737904 \\
\hline C5 & -0.25536367 \\
\hline $\mathrm{C} 6$ & 1.81681436 \\
\hline C7 & -1.85934190 \\
\hline C8 & 0.04378665 \\
\hline $\mathrm{C} 9$ & -0.12275135 \\
\hline C10 & -0.08196337 \\
\hline $\mathrm{C} 11$ & 0.39926550 \\
\hline C12 & -0.44249121 \\
\hline C13 & -0.16524689 \\
\hline C14 & -0.20848092 \\
\hline $\mathrm{H} 15$ & -1.90497648 \\
\hline $\mathrm{H} 16$ & -0.36941659 \\
\hline $\mathrm{H} 17$ & -1.31459261 \\
\hline $\mathrm{H} 18$ & -2.80954246 \\
\hline $\mathrm{H} 19$ & -1.96423011 \\
\hline $\mathrm{H} 20$ & -0.46756444 \\
\hline N21 & -1.020863062 \\
\hline 022 & -0.69962657 \\
\hline 023 & 0.65238010 \\
\hline 024 & 2.94564716 \\
\hline 025 & 0.08293386 \\
\hline 026 & -2.99140004 \\
\hline $\operatorname{Cr} 27$ & -0.01973447 \\
\hline C28 & 0.54075259 \\
\hline H2 9 & 1.03440265 \\
\hline $\mathrm{H} 30$ & 1.32314785 \\
\hline 131 & -0.0795689 \\
\hline
\end{tabular}

\section{angstroms}

Y

$-1.0982831525$

z

7.3667881541

$-0.1787193853$

3.7857498692

$-0.1397329900$

5.1543204615

$-2.0859756126$

5.2807866534

2.5239929796

$-4.4416749126$

$-4.5865571775$

$-0.3754210448$

0.1170855227

$-6.4604939888$

$-0.0078223493$

$-1.3730268628$

0.0225920291

$-2.6491147235$

$-1.7777042041$

$-4.5543342855$

1.9132642130

$-4.4900473858$

$-0.0397037270$

$-0.0590940748$

$-0.0729188620$

1.2106853203

$-1.8765720335$

4.2059876194

$-1.9907552436$

7. 6855521143

5.4149639350

5.7435758602

7.7847803206

7. 7865092619

5.9103446298

$-1.0249801390$

$-4.4283827978$

$-2.9149972441$

$-4.5325062190$

0.7844860728

$-4.3490393236$

0.1473514410

$-7.6271137307$

$-0.6527691826$

$-4.5836374673$

0.0688447663

$-4.5639008243$

0.9135097255

5.8556828187

1. 5420885648

5.1013357596

0.4570098321

6.4904039138

1. 5682538030

6.4964756318

\section{bond lengths (angstroms):}

$\begin{array}{llllll}\mathrm{C} 1 & -\mathrm{H} 16 & 1.108462 & \mathrm{C} 1 & -\mathrm{H} 19 & 1.105309 \\ \mathrm{C} 1 & -\mathrm{H} 20 & 1.097531 & \mathrm{C} 1 & -\mathrm{N} 21 & 1.460292 \\ \mathrm{C} 2 & -\mathrm{C} 3 & 1.369410 & \mathrm{C} 2 & -\mathrm{C} 5 & 1.265592 \\ \mathrm{C} 3 & -\mathrm{N} 21 & 1.370373 & \mathrm{C} 3 & -\mathrm{C} 28 & 1.518076 \\ \mathrm{C} 4 & -\mathrm{H} 15 & 1.099349 & \mathrm{C} 4 & -\mathrm{H} 17 & 1.107054 \\ \mathrm{C} 4 & -\mathrm{H} 18 & 1.104655 & \mathrm{C} 4 & -\mathrm{N} 21 & 1.463100\end{array}$




$\begin{array}{llllll}\text { C5 } & -\mathrm{C} 14 & 1.314665 & \mathrm{C} 6 & -\mathrm{O} 24 & 1.165350 \\ \mathrm{C} 6 & -\mathrm{Cr} 27 & 1.892804 & \mathrm{C} 7 & -\mathrm{O} 26 & 1.165541 \\ \mathrm{C} 7 & -\mathrm{C} 27 & 1.892628 & \mathrm{C} 8 & -\mathrm{O} 25 & 1.167669 \\ \mathrm{C} 8 & -\mathrm{Cr} 27 & 1.898270 & \mathrm{C} 9 & -\mathrm{C} 10 & 1.277102 \\ \mathrm{C} 9 & -\mathrm{C} 13 & 1.315006 & \mathrm{C} 10 & -\mathrm{Cr} 27 & 1.916355 \\ \mathrm{C} 11 & -023 & 1.165324 & \mathrm{C} 11 & -\mathrm{Cr} 27 & 1.893514 \\ \mathrm{C} 12 & -\mathrm{O} 22 & 1.165341 & \mathrm{C} 12 & -\mathrm{Cr} 27 & 1.893690 \\ \mathrm{C} 13 & -\mathrm{C} 14 & 1.270949 & \mathrm{C} 28 & -\mathrm{H} 29 & 1.099018 \\ \mathrm{C} 28 & -\mathrm{H} 30 & 1.106077 & \mathrm{C} 28 & -\mathrm{H} 31 & 1.106392\end{array}$

\section{bond angles:}

$\begin{array}{lllrllll}\mathrm{H} 19 & -\mathrm{C} 1 & -\mathrm{H} 16 & 108.025843 & \mathrm{H} 20 & -\mathrm{C} 1 & -\mathrm{H} 16 & 108.739903 \\ \mathrm{H} 20 & -\mathrm{C} 1 & -\mathrm{H} 19 & 108.505958 & \mathrm{~N} 21 & -\mathrm{C} 1 & -\mathrm{H} 16 & 110.756817 \\ \mathrm{~N} 21 & -\mathrm{C} 1 & -\mathrm{H} 19 & 109.426866 & \mathrm{~N} 21 & -\mathrm{C} 1 & -\mathrm{H} 20 & 111.299102 \\ \mathrm{C} 5 & -\mathrm{C} 2 & -\mathrm{C} 3 & 173.553841 & \mathrm{~N} 21 & -\mathrm{C} 3 & -\mathrm{C} 2 & 121.480898 \\ \mathrm{C} 28 & -\mathrm{C} 3 & -\mathrm{C} 2 & 119.520295 & \mathrm{C} 28 & -\mathrm{C} 3 & -\mathrm{N} 21 & 118.996378 \\ \mathrm{H} 17 & -\mathrm{C} 4 & -\mathrm{H} 15 & 109.087482 & \mathrm{H} 18 & -\mathrm{C} 4 & -\mathrm{H} 15 & 109.650247 \\ \mathrm{H} 18 & -\mathrm{C} 4 & -\mathrm{H} 17 & 108.625638 & \mathrm{~N} 21 & -\mathrm{C} 4 & -\mathrm{H} 15 & 109.285493 \\ \mathrm{~N} 21 & -\mathrm{C} 4 & -\mathrm{H} 17 & 110.606397 & \mathrm{~N} 21 & -\mathrm{C} 4 & -\mathrm{H} 18 & 109.569260 \\ \mathrm{C} 14 & -\mathrm{C} 5 & -\mathrm{C} 2 & 178.108578 & \mathrm{Cr} 27 & -\mathrm{C} 6 & -\mathrm{O} 24 & 179.131928 \\ \mathrm{Cr} 27 & -\mathrm{C} 7 & -\mathrm{O} 26 & 179.149312 & \mathrm{Cr} 27 & -\mathrm{C} 8 & -\mathrm{O} 25 & 179.970716 \\ \mathrm{C} 13 & -\mathrm{C} 9 & -\mathrm{C} 10 & 179.967213 & \mathrm{Cr} 27 & -\mathrm{C} 10 & -\mathrm{C} 9 & 179.964255 \\ \mathrm{Cr} 27 & -\mathrm{C} 11 & -\mathrm{O} 23 & 179.181056 & \mathrm{Cr} 27 & -\mathrm{C} 12 & -\mathrm{O} 22 & 179.188953 \\ \mathrm{C} 14 & -\mathrm{C} 13 & -\mathrm{C} 9 & 179.854186 & \mathrm{C} 13 & -\mathrm{C} 14 & -\mathrm{C} 5 & 179.850573 \\ \mathrm{C} 3 & -\mathrm{N} 21 & -\mathrm{C} 1 & 123.712759 & \mathrm{C} 4 & -\mathrm{N} 21 & -\mathrm{C} 1 & 114.892308 \\ \mathrm{C} 4 & -\mathrm{N} 21 & -\mathrm{C} 3 & 120.975429 & \mathrm{C} 7 & -\mathrm{Cr} 27 & -\mathrm{C} 6 & 176.982866 \\ \mathrm{C} 8 & -\mathrm{Cr} 27 & -\mathrm{C} 6 & 91.496863 & \mathrm{C} 8 & -\mathrm{Cr} 27 & -\mathrm{C} 7 & 91.520251 \\ \mathrm{C} 10 & -\mathrm{Cr} 27 & -\mathrm{C} 6 & 88.430762 & \mathrm{C} 10 & -\mathrm{Cr} 27 & -\mathrm{C} 7 & 88.552136 \\ \mathrm{C} 10 & -\mathrm{Cr} 27 & -\mathrm{C} 8 & 179.907333 & \mathrm{C} 11 & -\mathrm{Cr} 27 & -\mathrm{C} 6 & 90.710278 \\ \mathrm{C} 11 & -\mathrm{Cr} 27 & -\mathrm{C} 7 & 89.211035 & \mathrm{C} 11 & -\mathrm{Cr} 27 & -\mathrm{C} 8 & 91.285013 \\ \mathrm{C} 11 & -\mathrm{Cr} 27 & -\mathrm{C} 10 & 88.773794 & \mathrm{C} 12 & -\mathrm{Cr} 27 & -\mathrm{C} 6 & 89.252115 \\ \mathrm{C} 12 & -\mathrm{Cr} 27 & -\mathrm{C} 7 & 90.693493 & \mathrm{C} 12 & -\mathrm{Cr} 27 & -\mathrm{C} 8 & 91.242491 \\ \mathrm{C} 12 & -\mathrm{Cr} 27 & -\mathrm{C} 10 & 88.698739 & \mathrm{C} 12 & -\mathrm{Cr} 27 & -\mathrm{C} 11 & 177.472336 \\ \mathrm{H} 29 & -\mathrm{C} 28 & -\mathrm{C} 3 & 109.137955 & \mathrm{H} 30 & -\mathrm{C} 28 & -\mathrm{C} 3 & 111.687915 \\ \mathrm{H} 30 & -\mathrm{C} 28 & -\mathrm{H} 29 & 108.192204 & \mathrm{H} 31 & -\mathrm{C} 28 & -\mathrm{C} 3 & 111.618564 \\ \mathrm{H} 31 & -\mathrm{C} 28 & -\mathrm{H} 29 & 108.113786 & \mathrm{H} 31 & -\mathrm{C} 28 & -\mathrm{H} 30 & 107.967587\end{array}$

